\title{
785 nm Raman Spectroscopy of CVD Diamond Films
}

Paul William May, James A Smith, and Keith N Rosser

School of Chemistry, University of Bristol, Cantock's Close, Bristol, BS8 1TS, United Kingdom

\begin{abstract}
Raman spectroscopy is a powerful technique often used to study CVD diamond films, however, very little work has been reported for the Raman study of CVD diamond films using near infrared $(785 \mathrm{~nm})$ excitation. Here, we report that when using $785 \mathrm{~nm}$ excitation, the Raman spectra from thin polycrystalline diamond films exhibit a multitude of peaks (over 30) ranging from $400-3000 \mathrm{~cm}^{-1}$. These features are too sharp to be photoluminescence, and are a function of film thickness. For films $>30 \mu \mathrm{m}$ thick, freestanding films, and for films grown in diamond substrates the Raman peaks disappear, suggesting that the laser is probing the disordered small-grained interface between the diamond and substrate. Some of the peaks change in relative intensity with time ('blinking'), and the spectra are very sensitive to position on the substrate - this is reminiscent of the behaviour seen in SERS spectra..
\end{abstract}

\section{INTRODUCTION}

Raman spectroscopy is a powerful technique often used to study CVD diamond films $[1,2]$. Excitation using laser wavelengths in the UV and visible regions are most commonly used, and for diamond films these typically produce spectra with a limited number of characteristic peaks, including the diamond peak $\left(\sim 1332 \mathrm{~cm}^{-1}\right)$, the D and G bands from graphitic impurities, and sometimes the 1150 and $1450 \mathrm{~cm}^{-1}$ bands from $s p^{2}$ carbon impurities at the grain boundaries of nanophase diamond. Very little work has been reported, however, for the study of CVD diamond films using near infrared $(785 \mathrm{~nm}, 1.58 \mathrm{eV})$ excitation, which is exceptionally sensitive to $s p^{2}$ carbon. A few groups have used $785 \mathrm{~nm}$ excitation to study carbon films, but these have usually concentrated on studies of non-diamond carbon, such as measuring the dispersion of the $\mathrm{G}$ band in amorphous carbon films [3,4], carbon nanotubes and onions [5], or the graphitic residues following polishing of diamond [6]. It has also been used in combination with excitation from other wavelengths to study diamondoid molecules [7], revealing a multitude of peaks in the range $400-1500 \mathrm{~cm}^{-1}$ corresponding to the numerous different vibrations of the carbon framework in each molecule. It was suggested that the Raman spectrum from a sufficiently large diamondoid molecule would eventually resemble that seen for nanocrystalline diamond particles or films [8].

For CVD diamond films, however, the only Raman spectra reported using $785 \mathrm{~nm}$ excitation concerns the shift of the diamond line and Fano resonance effects during heavy boron doping [9], and some simple characterisation [10]. Very recently, Veres et al. [11] reported $785 \mathrm{~nm}$ Raman spectra from nanocrystalline diamond films with varying grain sizes, and found that samples with larger grains $(>125 \mathrm{~nm})$ exhibited spectra with a number of well distinguished sharp peaks. They suggested that this was due to the increased band gap of the larger crystals, which reduced the amount of resonantly enhanced $s p^{2}$ carbon structures allowing the remaining 
stronger peaks to become visible above the background. Previously [12], we reported that since the intensities of the diamond and non-diamond components in CVD diamond films vary as a function of excitation wavelength, multi-wavelength Raman studies could be used as a selective probe to study the of different constituents in the deposited films. In that paper we noted that unlike the other excitation wavelengths which gave the expected D, G and diamond peaks - the spectra obtained using $785 \mathrm{~nm}$ excitation gave a large number of well-defined peaks, and that the positions and intensities of these peaks changed in an unpredictable manner. This behaviour is unusual and unexpected, and we now report a series of experiments to attempt to elucidate the reasons behind this observation

\section{EXPERIMENT}

The CVD diamond films were taken from a variety of sources, however most were deposited using a conventional hot filament CVD reactor using a $1 \% \mathrm{CH}_{4} / \mathrm{H}_{2}$ process gas mixture at 20 Torr. The substrate temperature was constant at $\sim 900^{\circ} \mathrm{C}$ while the tantalum filament temperature was maintained at $2300 \mathrm{~K}$ (monitored by 2-colour optical pyrometer). The substrate was usually single crystal (100) silicon pre-abraded either manually or ultrasonically using 1$3 \mu \mathrm{m}$ diamond grit. The diamond growth rate was $\sim 0.5 \mu \mathrm{m} \mathrm{h}^{-1}$ and the film morphology was polycrystalline with the standard columnar growth structure typical of CVD diamond (see Fig.1).

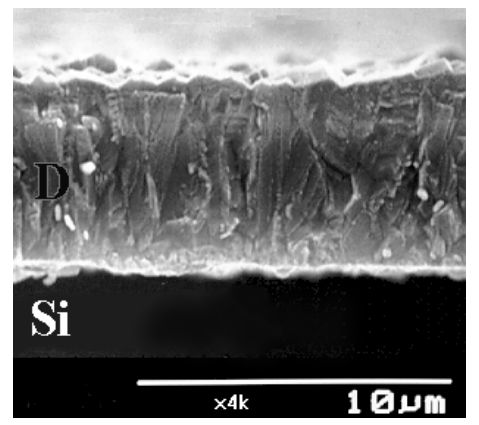

Figure 1. An electron micrograph cross-section through a $6 \mu \mathrm{m}$-thick HFCVD diamond film grown on Si. The diamond film (shown as 'D') has a columnar structure with an interface to the Si substrate consisting of a large number of disordered grains, small defective crystallites, and possibly SiC. Nearer the surface the film is predominantly microcrystalline diamond with relatively large crystallites and fewer grain boundaries.

The films were analysed using a Renishaw 2000 Raman spectrometer equipped with 3 laser wavelengths, the one of interest here being a $785 \mathrm{~nm}$ near-infrared diode laser. The resolution of the laser spot is $\sim 1 \mu \mathrm{m}$ and the sampling depth is estimated to be around a few $\mu \mathrm{m}$ depending upon the transparency and clarity of the diamond film. The accuracy of the calibration and run-to-run repeatability of the line positions was $\pm 0.2 \mathrm{~cm}^{-1}$ over the range 400$3000 \mathrm{~cm}^{-1}$. 


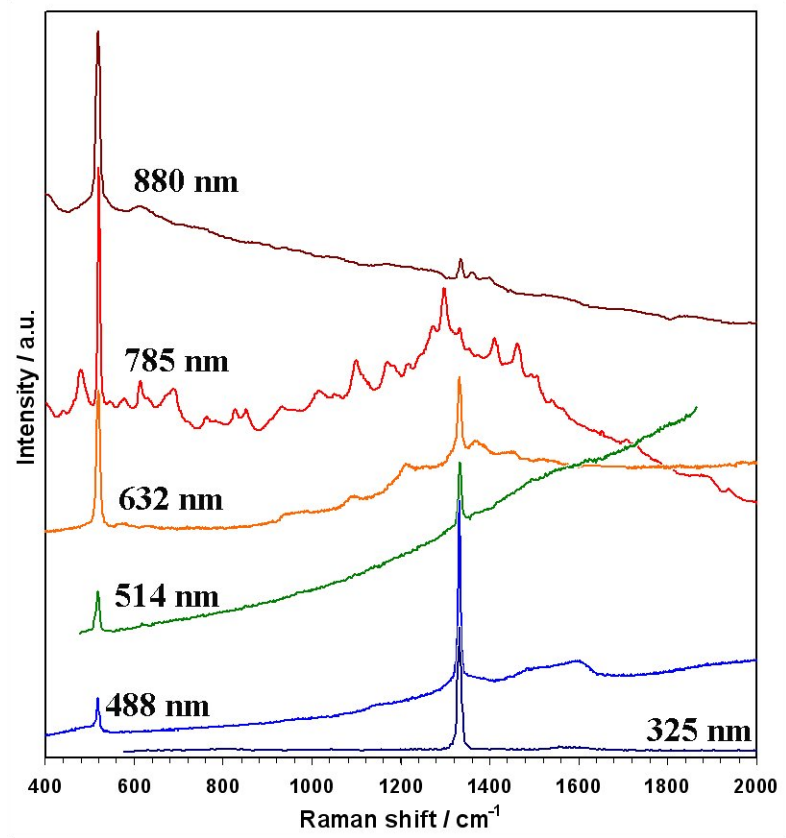

Figure 2. Raman spectra of a CVD diamond film grown for $4 \mathrm{~h}(\sim 2 \mu \mathrm{m}$ thick) on Si using different laser excitation wavelengths. The spectra have been scaled and offset vertically for ease of comparison. The peak at $520 \mathrm{~cm}^{-1}$ is due to the $\mathrm{Si}$ substrate, and other obvious features are the diamond line at $1332 \mathrm{~cm}^{-1}$, the $\mathrm{D}$ band at $\sim 1380 \mathrm{~cm}^{-1}$ and the $\mathrm{G}$ band $\sim 1580 \mathrm{~cm}^{-1}$.

Table 1. The following is a list of $785 \mathrm{~nm}$ Raman peak positions obtained from various CVD diamond films on Si substrates. The first-order $\left(520 \mathrm{~cm}^{-1}\right)$ and second-order (950$1000 \mathrm{~cm}^{-1}$ ) Si peaks are not included. Unless stated otherwise, these peaks are unassigned, but they probably originate from $s p^{2}$ carbon structures, defective diamond or nanophase carbon species present at the interface. Peaks shown with a range of positions (e.g. 2210$2213 \mathrm{~cm}^{-1}$ ) indicate that a sharp peak was seen at different positions within this range from a number of spectra taken from different samples, and so we have assumed that this is the same peak that shifts by a few $\mathrm{cm}^{-1}$. Values enclosed in square brackets have been given the same tentative assignment. The values which are underlined are those which have a superficial correspondence (within a few $\mathrm{cm}^{-1}$ ) to Raman features reported in the literature (and collated in Zaitsev's book [14]) and, therefore, may have a tentative assignment.

Peak positions $/ \mathbf{c m}^{-1}:$ 2223, 2210-2213, 2172, 2134, 2056, 2017, 1940, 1901, 1877, 1756, $1711,1675,1654-1658, \underline{1630}, \underline{1620}, \underline{1568},[1576, \underline{1540-1542]}$ ( $\mathrm{G}$ band?), 1507, 1500, [1467-1472, 1459-1465 1449, 1433-1436] (trans-polyacetylene?), 1420, 1412-1416, 1400, [1356-1359, 1350-1352] (D band?), 1331-1333 (diamond), [1323, 1311-1314] (lonsdaleite?), 1296-1299, 1271-1275, 1262-1264, 1243-1245, 1212-1220, 1185, 11691175, 1125-1134 (trans-polyacetylene?), 1116, 1098-1105, 1050, 1045, 1034, 1021-1026, 1009-1015, 1000, 976-985, 929-934, $\underline{897}, 890,878-883,852-853,835-841,828,783-785$, 760-764, 744-746, 713-718, 689-693, $\underline{655}, 645-649,630-634, \underline{625}, 613-616,597,577,570$, 561-563, 547-549, 537, 521-523, 495, 481-485, 465, 457, 438-443, 423, 405-407. 


\section{RESULTS}

Figure 2 shows a series of Raman spectra using different laser excitation wavelengths, taken from a standard $2 \mu \mathrm{m}$-thick CVD diamond film grown on Si. Most of the spectra show the expected peaks and bands from CVD diamond, but with varying intensities due to the different resonance efficiencies of the laser light with $s p^{2}$ or $s p^{3}$ carbon structures. However, the spectrum taken with $785 \mathrm{~nm}$ excitation shows substantially more structure than the other spectra, with upwards of 30 distinct peaks/features visible in the range shown. A listing of these peaks is given in Table 1. Thus, $785 \mathrm{~nm}$ excitation seems to be quite special in terms of its interaction of the diamond film compared to the other wavelengths - even those only $100 \mathrm{~nm}$ higher or lower in wavelength - and it appears to substantially enhance features that are normally too small to see.

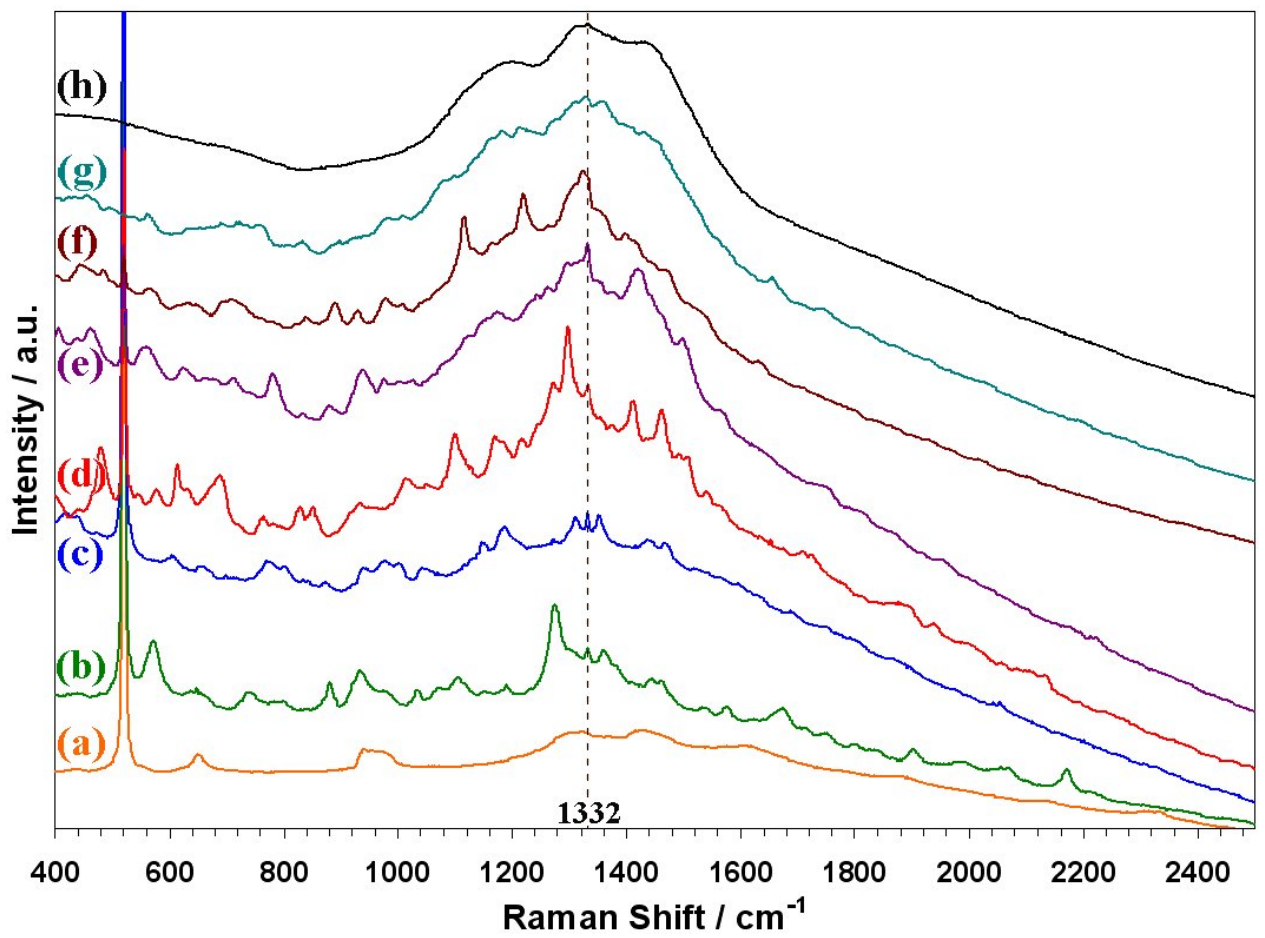

Figure 3. Raman spectra (785 nm excitation) of CVD diamond films grown on Si using the HF reactor (Ta filament) for varying deposition times, and hence thicknesses. (a) 20 mins, $0.17 \mu \mathrm{m}$, (b) 45 mins, $0.38 \mu \mathrm{m}$, (c) $1.5 \mathrm{~h}, 0.75 \mu \mathrm{m}$, (d) $4 \mathrm{~h}, 2 \mu \mathrm{m}$, (e) $10 \mathrm{~h}, 5 \mu \mathrm{m}$, (f) $17 \mathrm{~h}, 8.5 \mu \mathrm{m},(\mathrm{g}) 32 \mathrm{~h}, 16 \mu \mathrm{m}$, (h) $100 \mathrm{~h}, 50 \mu \mathrm{m}$. The feature at $950-1000 \mathrm{~cm}^{-1}$ in (a) is the second-order peak from the Si substrate. The spectra have been scaled and offset vertically for clarity. The dashed vertical line marks the position of the diamond line at $1332 \mathrm{~cm}^{-1}$.

Figure 3 shows the $785 \mathrm{~nm}$ Raman spectra for a series of HFCVD films of varying thicknesses. For films $<1 \mu \mathrm{m}$ thick, there are few peaks and little structure in the spectrum, which is dominated by the large peak from the Si substrate. As the film thickness increases above $1 \mu \mathrm{m}$, the spectra become more complicated, with a multitude of peaks appearing. These peaks and features behave in an unpredictable way: some appear in all spectra, some only in a 
few of the spectra, and most change relative intensities between spectra in a seemingly random way. The peaks reach a maximum in terms of quantity and complexity at a film thickness of between 2-5 $\mu \mathrm{m}$. For films thicker than $5 \mu \mathrm{m}$, the number of distinct peaks decreases until by a thickness of $\sim 50 \mu \mathrm{m}$ they have disappeared altogether, and the spectrum just consists of a few broad humps (centred $\sim 1200, \sim 1330$, and $\sim 1420 \mathrm{~cm}^{-1}$ ) on a large photoluminescent background.

A series of experiments were performed to try to pin down the cause of these Raman lines. First, $2-\mu \mathrm{m}$-thick diamond films were grown in the same reactor under identical conditions using filaments made from tungsten and rhenium. The $785 \mathrm{~nm}$ Raman spectra from each of these films was identical to the spectrum from the film grown with a tantalum filament (Fig.2). This indicates that the origin of the peaks is unlikely to be contamination in or on the diamond surface from filament material. To be certain, these films were then treated with concentrated $10 \%$ nitric acid/sulphuric acid mixture for $24 \mathrm{~h}$, which should dissolve away any metallic residues on the surface. After this treatment the spectra were unchanged. We used similar conditions in a microwave plasma reactor to grow a CVD diamond film of similar thickness and morphology to the ones grown under HFCVD. The Raman spectrum from this MW film also exhibited the Raman features (as was noted previously [12]), which rules out contamination effects from our HF system as being the origin of the peaks.

To rule out substrate effects, films were grown on a variety of substrate materials using identical conditions to before. $2-\mu \mathrm{m}$-thick films were grown on tungsten, tungsten carbide, aluminium nitride, quartz, molybdenum, n-type $\mathrm{Si}$, p-type $\mathrm{Si}$, and undoped (intrinsic) Si, and these all exhibited $785 \mathrm{~nm}$ Raman spectra containing a multitude of peaks, although as before, the relative intensities of the peaks was variable. The exception to this was for films grown on HPHT single crystal (100) diamond substrates. Since the films were not grown under optimum homoepitaxial growth conditions, deposition formed a polycrystalline layer, although since the substrate was diamond there was no nucleation layer consisting of nanocrystalline diamond. The $785 \mathrm{~nm}$ Raman spectrum from this film gave only the diamond zone-centre line, with no other features present in the spectrum (see Fig.4).

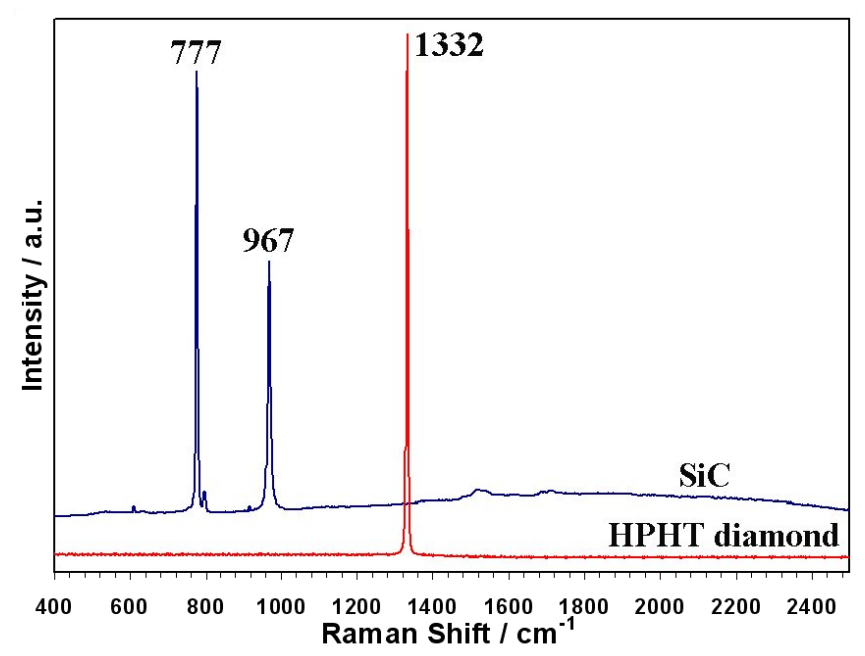

Figure 4. $785 \mathrm{~nm}$ Raman spectrum of single crystal silicon carbide, and that of a $2 \mu \mathrm{m}$ CVD diamond polycrystalline film grown on a HPHT single crystal diamond substrate. 
One possibility was that the peaks could be due to SiC layers or nanocrystals at the diamond/Si interface. This is because $785 \mathrm{~nm}$ excitation has been shown to enhance the Raman scattering from nitrogen defects in $\mathrm{SiC}$ [13] giving rise to extra peaks. Figure 4 shows the $785 \mathrm{~nm}$ Raman spectrum of single crystal SiC taken with our system under identical conditions to before. The spectrum is dominated by two peaks at 777 and $967 \mathrm{~nm}$, with only a few very minor features elsewhere. Thus, if the features seen in the diamond spectra were due to $\mathrm{SiC}$, we should also observe these two intense characteristic peaks. Since these 2 peaks are not present, we can conclude that the features are not due to $\mathrm{SiC}$ - and this is confirmed by the fact that the features are present when using non-Si substrates.

An interesting observation was that freestanding diamond films that had delaminated from the substrate did not show any of the Raman features, and just gave a broad PL hump, similar to that seen in Fig.3(h). This was true for thick $(150 \mu \mathrm{m})$ and thin $(4 \mu \mathrm{m})$ freestanding films, and indicates that the Raman peaks we see require the presence of a diamond film bonded directly onto a substrate surface.

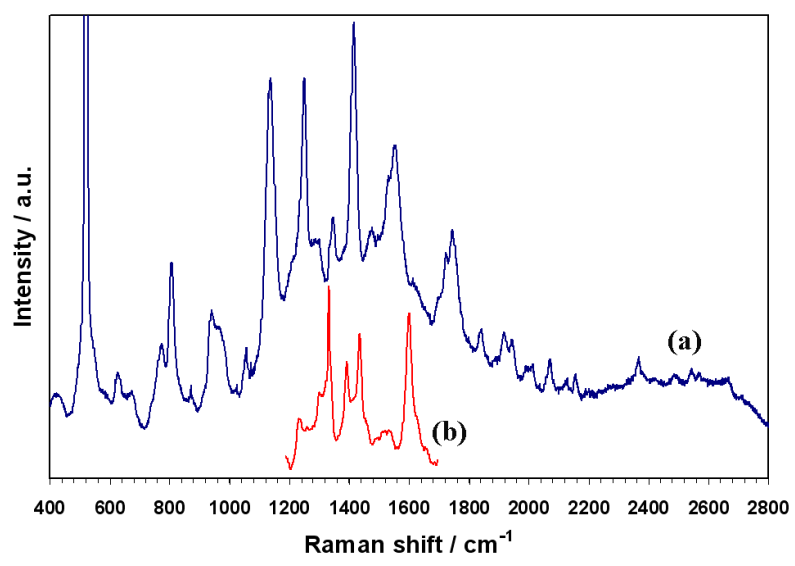

Figure 5. $785 \mathrm{~nm}$ Raman spectrum of diamond film grown for $4 \mathrm{~h}(\sim 2 \mu \mathrm{m}$ thick $)$ on $\mathrm{Si}$ with different surface pre-treatments prior to deposition: (a) seeded with $0.1 \mu \mathrm{m}$ diamond powder, (b) smeared with hydrocarbon oil.

The effect of nucleation was then studied by performing a series of experiments with different abrasion/pre-treatment methods. We saw, above, that for 'perfect' nucleation (as occurs on a diamond substrate), the Raman peaks were not present. Manual abrasion of a Si substrate using $0.1 \mu \mathrm{m}$ diamond powder (compared to 1-3 $\mu \mathrm{m}$ powder) made no significant difference to the type of diamond film that was grown, or to the $785 \mathrm{~nm}$ Raman spectrum it exhibited. Similarly, ultrasonic abrasion using either $1-3 \mu \mathrm{m}$ or $0.1 \mu \mathrm{m}$ diamond/water slurry gave the same results. However, seeding the surface (but with no abrasion) with a submonolayer of $0.1 \mu \mathrm{m}$ diamond powder followed by $4 \mathrm{~h}$ growth produced a film with very high nucleation density and a highly structured Raman spectrum (see Fig.5(a)). Smearing hydrocarbon rotary-pump oil onto the $\mathrm{Si}$ wafer, followed by $4 \mathrm{~h}$ growth produced isolated islands of diamond growth (with no residue remaining of the hydrocarbon oil). The Raman spectra from these islands gave rise to Raman spectra with an extremely large enhancement for some peaks (see Fig.5(b)). Note that the positions of many of these enhanced peaks in Fig.5(b) are significantly different to the ones observed in Fig.4(a), which means that different peaks are being enhanced in each case. 
We observed that if the same area of a sample was analysed repeatedly over a period of a few hours, the relative intensities of some of the peaks would fluctuate from spectrum-tospectrum. This fluctuation would sometimes be substantial, with peaks disappearing, and sometimes reappearing again ('blinking') or new peaks emerging. Sampling from several different positions that were only a few $100 \mu \mathrm{m}$ apart on the same film also gave rise to spectra that were very different from each other. Some peaks would be common to all spectra, and some peaks would appear in some, or only one of the spectra. Peaks often appeared to have shifted by as much as $5 \mathrm{~cm}^{-1}$ in either direction, although it was unclear whether this was the same peak or a new one that had emerged while the previous one had disappeared.

\section{CONCLUSIONS}

We have seen that the Raman spectra of thin diamond films obtained using $785 \mathrm{~nm}$ excitation appear very different to those obtained using other wavelengths, and that the spectra consist of a multitude (at least 30 in the range $400-3000 \mathrm{~cm}^{-1}$ ) of sharp, well defined peaks which fluctuate greatly in intensity ('blink') as a function of time and position on the substrate. These features are too sharp to be photoluminescence [14], and are a function of film thickness. For films $>30 \mu \mathrm{m}$ thick, for freestanding films, and for films grown on a diamond substrate, these Raman peaks disappear, suggesting that the peaks originate from the disordered small-grained material at the interface between the diamond and substrate. Since the peaks are independent of substrate material, metals, $\mathrm{SiC}$ and other metal carbides or impurities can be discounted, leaving $s p^{2}$ and $s p^{3}$ carbon structures as prime candidates. Possible structures responsible for some of these peaks include:

(i) Polymorphs of diamond, such as lonsdaleite (or hexagonal diamond), which is reported to exhibit a sharp Raman peak appearing between $1315 \mathrm{~cm}^{-1}$ and $1326 \mathrm{~cm}^{-1}$ [15]. Such features are reported to have been observed on cut (polished) diamond surfaces, on diamond surfaces prepared using thermochemical polishing with hot transition metals, and around indentations made on diamond surfaces [16].

(ii) Isolated diamond nanocrystals of varying sizes $(1 \mathrm{~nm}-100 \mathrm{~nm})$ can exhibit a zonecentre phonon line ranging from $1332 \mathrm{~cm}^{-1}$ down to $1315 \mathrm{~cm}^{-1}$ as the crystal size decreases as a result of heating by the laser to temperatures as much as $700 \mathrm{~K}[17,18]$. It is highly likely that these nanocrystals such as these would be present at or near the interface, however, the high thermal conductivity of the surrounding diamond film should efficiently spread the heat away maintaining the local temperature at near ambient. Alternatively, stress is known to shift the position of the diamond line or split it into 2 or 3 components in the range $1328-1370 \mathrm{~cm}^{-1}$ [14]. So the peaks we observe could be due to a variety of diamond nanocrystals experiencing varying amounts of compressive or tensile stress. Again, we feel this is unlikely, as stress effects tend to shift the diamond line by only a few $\mathrm{cm}^{-1}$, whereas we observe peaks hundreds of $\mathrm{cm}^{-1}$ away from $1332 \mathrm{~cm}^{-1}$.

(iii) Diamondoid molecules may also be present at the interface, and these are known to exhibit a multitude of Raman peaks between $400-3000 \mathrm{~cm}^{-1}[7,8]$.

(iv) Defects and impurities (nitrogen, silicon) often give rise to Raman peaks in diamond, and these peaks are comprehensively catalogued in the book by Zaitsev [14]. Some of the peaks observed in this work can be matched (within a few $\mathrm{cm}^{-1}$ ) with some of 
those reported in Zaitsev's book, and a tentative assignment suggested. However, I would treat such assignments with caution until corroborating evidence can be found.

(v) Resonant Raman scattering from a variety of $s p^{2}$ carbon structures present at the grain boundaries could account for the high sensitivity.

Without further information, it is not possible at this stage to state which of the above (if any) are responsible for the Raman features we see. However, another question remains: why are we able to see these features at all? These Raman lines are often as intense or more intense than the diamond zone-centre line, which is very surprising, given the relatively large amount of material present in the several $\mu \mathrm{m}$ of diamond film compared to that present at the interface. This suggests that we are witnessing a substantial enhancement of the Raman signal from those species which are at or near the surface/interface. Such enhancement of Raman signals is not unknown - indeed, it is the basis of the surface enhanced Raman spectroscopy (SERS) technique [19]. Furthermore, one well known feature of SERS is the instability of the spectra, with peaks 'blinking' in intensity with time [20]. Experiments to deliberately take SERS spectra from diamond nanoparticles [21] and films [22,23,24] involve many preparation steps and are difficult to perform, but yield spectra that look very similar to ours, with a plethora of sharp peaks in the range $400-3000 \mathrm{~cm}^{-1}$ that been assigned as various types of unspecified $s p^{2}$ carbon species. But SERS relies on the excitation of surface-plasmon resonances in metal (usually Ag) films or nanoparticles that have been evaporated onto the surface, which then efficiently couple energy into the nearby film or particle of interest, resulting in huge enhancement of its Raman signal. CVD diamond films have no metallic impurities present on the surface - and this was ensured by acid dips before and after abrasion. If so, how can our observations be consistent with the SERS effect? One possible explanation is that the disordered nanocrystalline film found at the nucleation layer at the interface could be considered to be similar to nanodiamond particles with a graphitic or a-C coating. If this conducting coating had, by coincidence, a similar surface plasmon energy to that of $785 \mathrm{~nm}$ light, then excitation at this wavelength would produce a SERS-like effect. However, this mechanism is still conjecture at this time.

Whether or not a SERS-like effect is the correct explanation for the observed Raman signal enhancement, we have shown that $785 \mathrm{~nm}$ Raman can be a useful tool to study CVD diamond films, and especially the diamond/surface interface

\section{ACKNOWLEDGMENTS}

The authors would like to thank Jacob Filik for many useful discussions.

\section{REFERENCES}

1. J. Filik, Spectroscopy Europe 17, (2005) 10.

2. S. Prawer, R.J. Nemanich, Phil. Trans. R. Soc. Lond. A 362, (2004) 2537.

3. M. Koós, M. Veres, S. Tóth, M. Füle, Raman spectroscopy of CVD carbon thin films excited by near-infrared light, in: G. Messina, S. Santangelo (Eds.), Carbon: The Future Material for Advanced Technology Applications, Springer' Series Topics in Applied Physics, 100 (2005), p. 423 (Springer, Berlin, 2005).

4. A.C. Ferrari, J. Robertson, Phil. Trans. R. Soc. Lond. A 362, (2004) 2477 
5. D. Roy, Manish Chhowalla, H. Wang, N. Sano, I. Alexandrou, T.W. Clyne, G.A.J. Amaratunga, Chem. Phys. Lett. 373, (2003) 52.

6. J.R. Hird, M. Bloomfield, I.P. Hayward, Phil. Mag. 87, (2007) 267

7. J. Filik, J.N. Harvey, N.L. Allan, P.W. May, J.E.P. Dahl, S. Liu, and R.M.K. Carlson, Spectrochimica Acta A: Mol. Biomol. Spect. 64, (2006) 681.

8. J. Filik, J.N. Harvey, N.L. Allan, P.W. May, J.E.P. Dahl, S. Liu, R.M.K. Carlson, Phys. Rev. B. 74, (2006) 035423.

9. M. Pedroza-Montero, V. Chernov, B. Castañeda, R. Meléndrez, J. A. N. Gonçalves, G.M. Sandonato, R. Bernal, C. Cruz-Vázquez, F. Brown, E. Cruz-Zaragoza, M. Barboza-Flores, Phys. Stat. Sol. (a) 202, (2005) 2154.

10. N.M. Balzaretti, A. Feldman E.S. Etz, R. Gat, J. Mater. Res. 14, (1999) 3720.

11. M. Veres, S. Tóth, M. Füle, M. Koós, abstract book from 'New Diamond and NanoCarbons' conference, Osaka Japan, 2007.

12. S.M. Leeds, T.J. Davis, P.W. May, C.D.O. Pickard and M.N.R. Ashfold, Diamond Relat. Maters. 7, (1998) 233.

13. J.C. Burton, F.H. Long, I.T. Ferguson, J. Appl. Phys. 86, (1999) 2073.

14. A.M. Zaitsev, Optical Properties of Diamond: A Data Handbook (Springer, Berlin, 2001).

15. D.S. Knight and W.B. White, J. Mater. Res. 4, (1989) 385.

16. Y.G. Gogotsi, A. Kailer and K.G. Nickel, J. Appl. Phys. 84, (1998) 1299.

17. X.-Z. Zhao, K.A. Cherian, R. Roy, W.B. White, J. Mater. Res. 13, (1998) 1974.

18. P.W. May, P. Overton, J.A. Smith, K.N. Rosser, MRS Symp. Proc. (Fall 2007), in press.

19. Selected Papers on Surface-Enhanced Raman Scattering, edited by. M. Kerker, SPIE Milestone Series MS 10, (Spie Billingham, Wa, USA, 1990).

20. M. Veres, M. Fule, S. Toth, M. Koos, I. Pocsik, Diamond Relat. Mater. 13, (2004) 1412.

21. E. Perevedentseva, A. Karmenyan, P.-H. Chung and C.-L. Cheng, J. Vac. Sci. Technol. B, 23, (2005) 1980.

22. D.S. White, R. Weimer, L. Pilione, W.B. White, Appl. Phys. Lett. 56, (1990) 1320.

23. D. Roy, Z.H. Barber, T.W. Clyne, J. Appl. Phys. 91, (2002) 6085.

24. T. López-Ríos, É. Sandré, S. Leclercq, and É. Sauvain, Phys. Rev. Lett. 76, (1996) 4935. 